Short Research Communication

\title{
Human Cartilage Engineering in an In Vitro Repair Model Using Collagen Scaffolds and Mesenchymal Stromal Cells
}

\author{
Clara Sanjurjo-Rodríguez ${ }^{1,3}$, Rocío Castro-Viñuelas ${ }^{1,2}$, Tamara Hermida-Gómez ${ }^{2,3}$, Isaac Manuel \\ Fuentes-Boquete1,3, Francisco Javier de Toro1,3, Francisco Javier Blanco ${ }^{2,3}$, Silvia María Díaz-Prado1,3凶 \\ 1. Cell Therapy and Regenerative Medicine Unit, Rheumatology Group, Institute of Biomedical Research of A Coruña (INIBIC), University Hospital Complex \\ A Coruña (CHUAC), Galician Health Service (SERGAS), Department of Medicine, Faculty of Health Sciences, University of A Coruña, A Coruña, Spain. \\ 2. Tisular Bioengineering and Cell Therapy Unit (GBTTC-CHUAC), Rheumatology group, Institute of Biomedical Research of A Coruña (INIBIC), University \\ Hospital Complex A Coruña (CHUAC), Galician Health Service (SERGAS), A Coruña, Spain. \\ 3. CIBER de Bioingeniería, Biomateriales y Nanomedicina (CIBER-BBN) \\ \ Corresponding author: Tel. +34 9811763 99, e-mail: s.diaz1@udc.es, silvia.ma.diaz.prado@sergas.es \\ (c) Ivyspring International Publisher. This is an open access article distributed under the terms of the Creative Commons Attribution (CC BY-NC) license \\ (https://creativecommons.org/licenses/by-nc/4.0/). See http://ivyspring.com/terms for full terms and conditions.
}

Received: 2017.02.27; Accepted: 2017.08.07; Published: 2017.09.28

\begin{abstract}
The purpose of this study was to investigate cartilage repair of in vitro lesion models using human bone marrow mesenchymal stromal cells (hBMSCs) with different collagen (Col) scaffolds.

Lesions were made in human cartilage biopsies. Injured samples were pre-treated with interleukin $1 \beta$ (ILI $\beta$ ) for $24 \mathrm{~h}$; also, samples were not pre-treated. hBMSCs were seeded on different types of collagen scaffolds. The resulting constructs were placed into the lesions, and the biopsies were cultured for 2 months in chondrogenic medium.

Using the modified ICRSII scale, neotissues from the different scaffolds showed ICRS II overall assessment scores ranging from $50 \%$ (fibrocartilage) to $100 \%$ (hyaline cartilage), except for the Col I +Col II +HS constructs (fibrocartilage/hyaline cartilage, 73\%). Data showed that hBMSCs cultured only on Col I +Col II +HS scaffolds displayed a chondrocyte-like morphology and cartilage-like matrix close to native cartilage. Furthermore, ILI $\beta$ pre-treated biopsies decreased capacity for repair by hBMSCs and decreased levels of chondrogenic phenotype of human cartilage lesions.
\end{abstract}

Key words: Regenerative Medicine, Tissue Engineering, Hyaline Cartilage, Tissue Scaffolds, Mesenchymal Stromal Cells, Osteoarthritis

\section{Introduction}

It is widely accepted that three-dimensional (3D) in vitro cultures simulate the in vivo situation better than two-dimensional ones. Although micromass culture is considered a useful tool for studies of chondrogenesis, it is not suitable for cell therapy [1]. Scaffolds can, however, provide an appropriate 3D environment for cell viability and proliferation, cell differentiation and maintenance of a specific phenotype [2].

Tissue Engineering using biodegradable scaffolds, cells and cell factors has evolved into a multidisciplinary field with an aim to develop biological substitutes with biochemical, structural, morphological and functional properties similar to native cartilage for subsequent use for in vivo treatment [3]. The most important issue in Tissue Engineering is the selection of cell type and optimal scaffold.

Most studies have tested growth and differentiation of cells on various scaffolds but have not placed the constructs inside a native cartilage environment or taken the host tissue into account [4]. In vitro testing is easier than in vivo testing for obtaining standardized and quantifiable information about cell cytotoxicity, proliferation and differentiation capacity [5]. This model allows us to 
analyze human cartilage samples from a single donor using different study variables and to test different constructs in an in vitro native environment. In our study, this model was used to test the repair capacity of different constructs.

\section{Materials and Methods}

\section{Isolation and culture of bone marrow stromal cells}

Each donor in the study gave informed consent according to the guidelines of the local ethics committee and principles of Declaration of Helsinki. This study was approved by the Ethics Committee of Clinical Investigation of Galicia (Santiago de Compostela, Spain).

Bone marrow cells were obtained from three hip fracture patients who underwent total hip replacement, as previously described [6]. Cells were extracted by washing the bone marrow with culture medium: Dulbecco's Modified Eagle's Medium (DMEM; Lonza, Spain) with 20\% foetal bovine serum (LabClinics, Spain). The isolated cells were cultured in a $5 \%$ humidified $\mathrm{CO}_{2}$ atmosphere at $37^{\circ} \mathrm{C}$. The resultant cell suspension was cultured and expanded. We previously confirmed by characterization [6] that cells obtained by this method are hBMSCs.

\section{Scaffolds}

Different Col scaffolds (Opocrin S.p.A., Modena, Italy) had been previously tested by our group [6, 7]. From those, scaffolds producing better chondrogenic hBMSC phenotypes, including type I collagen (Col I), Col I and heparan sulfate (Col I+HS), Col I and type II collagen (Col II) and HS (Col I+Col II+HS) and Col I and $\mathrm{Col}$ II and chondroitin sulfate ( $\mathrm{Col} \mathrm{I}+\mathrm{Col} \mathrm{II}+\mathrm{CHS})$ were selected. The hBMSCs were cultured on the surface of $4 \mathrm{~mm}$-diameter sponges and incubated for 1 $\mathrm{h}$ at $37^{\circ} \mathrm{C}$. These constructs were used for the in vitro lesion model using chondrogenic medium: hMSC Chondrogenic Differentiation Bulletkit medium (Lonza, Spain) with 10ng/ $\mathrm{ml}$ of human transforming growth factor $\beta-3$ (TGF $\beta-3$, Prospec-Tany Technogene Ltd., Israel).

\section{In vitro lesion model}

Cartilage samples were obtained from three patients who underwent total hip or knee replacement because of fracture or osteoarthritis (OA). The cartilage was sliced with a scalpel and $6 \mathrm{~mm}$ biopsies were obtained using disposable biopsy punches (Kai Medical, Germany).

$3 \mathrm{~mm}$-Diameter lesions were made in human cartilage biopsies using a dental drill (Gebr. Brasseler Gmbh \& Co. KQ, Germany) with a rotor (EWL K9,
Germany). Over a two-month period, two experiments were performed:

1) Non-IL1 $\beta$ Group: $2 \times 10^{5}$ cells were seeded on scaffolds. These constructs were placed into the lesion and chondrogenic medium was added. The medium was changed every 3-4 days.

2) IL1 $\beta$ Group: Cartilage biopsies with lesions were pre-treated with $10 \mathrm{ng} / \mathrm{ml}$ of IL1 $\beta$ (Sigma, USA) for 24 hours. $2 \times 10^{5}$ cells were seeded on scaffolds, the resulting sponge-constructs were introduced into the lesion and chondrogenic medium was added. The medium was changed every 3-4 days.

Following controls were included: defect without constructs, defect with scaffolds and without cells, and native cartilage without defect (Figure S1).

\section{Histological analysis}

The resulting neotissue was evaluated histochemically using $4 \mu \mathrm{m}$-thick deparaffinized sections. These sections were then stained with hematoxylin and eosin (HE), and stained for collagen and proteoglycans (PGs) with Masson's trichrome (MT) or Safranin O (SO), respectively. The stained sections were examined using an optical microscope (Olympus BX61, USA) equipped with a digital camera (Olympus DP70, USA). Quantitative analyses of the SO staining were measured using ImageJ $1.48 \mathrm{v}$ (National Institutes of Health, Bethesda, USA). After the colour substraction of non-stained areas, the percentage of metachromatic areas was measured and expressed as mean \pm standard error.

To assess the quality of cartilage repair, the ICRS II histological scoring system [8] was used with some modifications to adapt it for analysis of in vitro cartilage repair. The neotissues formed were scored by three blind observers. Our modified score system comprised 7 out of the 14 parameters: "cell morphology" and "chondrocyte clustering" as well as "surface architecture" and the "basal integration" of the neotissue formed were observed by the HE staining; "tissue morphology" was assessed by analyzing the collagen fiber distribution with MT staining; the parameter to evaluate PG content was "matrix staining", which was assessed using SO staining; and, the "overall assessment" was the statistical mean of the values for all 6 parameters analyzed.

Further immunohistochemical analysis of Col I, Col II and aggrecan (Agg) was performed in samples with highest ICRS II score.

\section{Results}

\section{Controls and Non-ILI $\beta$ model}

Control constructs without cells did not show 
presence of neotissue formation and the scaffolds were not degraded (Figure S1, Ctrl w/o cells). Self-repair did not occur in the negative control (Figure S1, control lesion). In the Non-IL1 $\beta$ model, histochemistry after two months revealed that cells seeded on all scaffolds filled almost $100 \%$ of the lesion and that neotissues were integrated with the border of the lesion (Figure 1, H-E Non-IL). Scaffolds were almost fully degraded in this model, except the Col I+HS scaffold, and cellular density in all the grafts was higher (Figure 1, H-E Non-IL) than that in the native cartilage (Figure S1, positive control). Spindle- and round-shaped cell morphology was found in all neotissues.

Staining with MT showed the presence of $\mathrm{Col}$ in the ECM of all the constructs (Figure 1, H-E Non-IL), that was observably more homogeneous in the tissues of the $\mathrm{Col} \mathrm{I}+\mathrm{Col} \mathrm{II}+\mathrm{HS}$ constructs. The presence of PGs detected by SO staining (Figure 1, H-E Non-IL) was more metachromatic in the Col I+Col II+HS constructs $(52.426 \pm 4.877)$ than in Col I $(5.107 \pm 3.337)$, Col I+HS (23.406 \pm 6.189$)$ and $\mathrm{Col} \mathrm{I+Col} \mathrm{II+CHS}$ (32.722 \pm 8.781$)$ constructs.

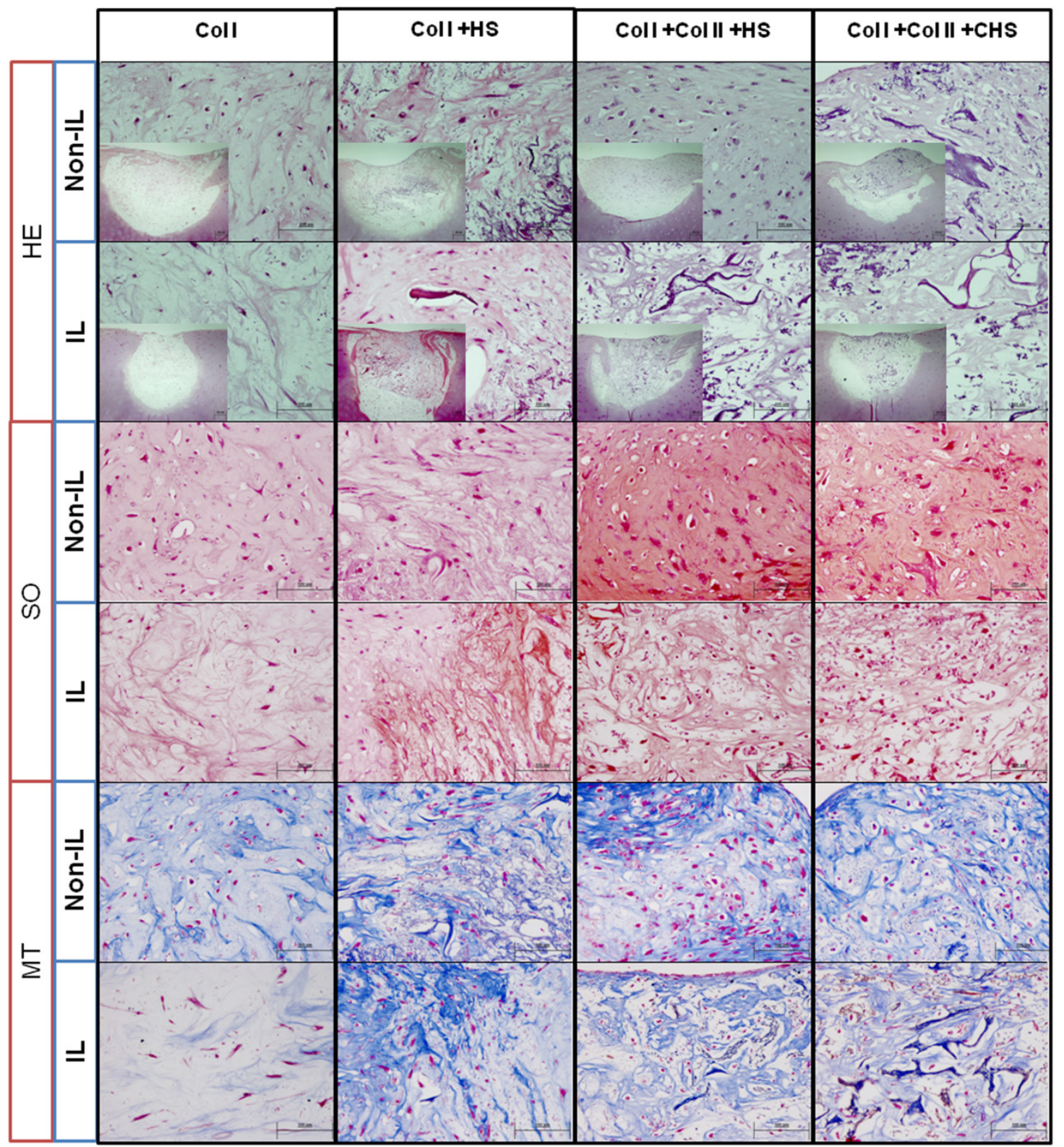

Figure 1. Histology of repair in human cartilage non interleukin- $1 \beta$-treated lesions (Non-IL) and IL $1 \beta$-treated lesions (IL). Hematoxylin-eosin (HE), Safranin O (SO) and Masson's Thricrome (MT) staining of the models. Scale bar 100 $\mu \mathrm{m}$ (smaller images $200 \mu \mathrm{m}$ ). Col I: type I collagen; Col II: type II collagen; HS: heparan sulfate; CHS: chondroitin sulfate. 


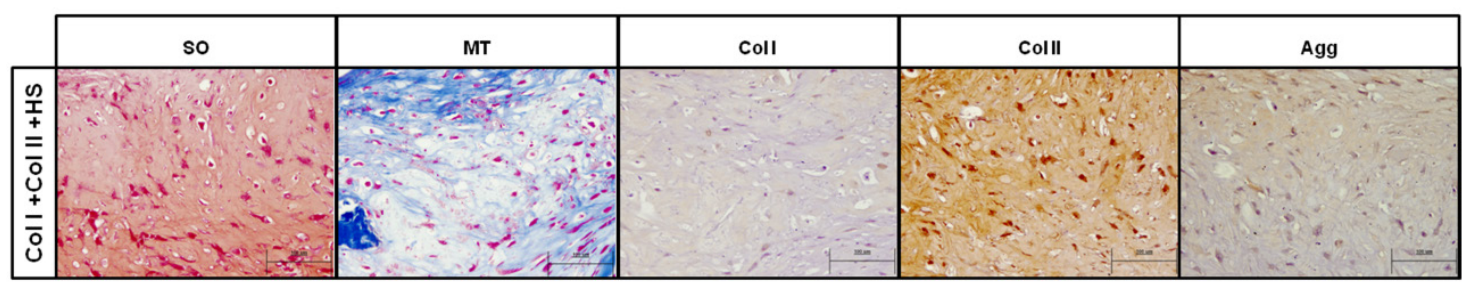

Figure 2. Histology and immunohistochemistry of repair obtained using type I and II collagen and heparan sulfate scaffolds (Col I+Col II+HS), in non-interleukin-1 ß-treated lesions. Images of Safranin O (SO) and Masson's Thricrome (MT) staining, and type I (Col I) and II (Col II) collagen and aggrecan (Agg) immunostaining performed on the neotissues formed on Col I+Col II+HS scaffolds in the Non-IL model. Col and Agg immunostaining were counterstaining with hematoxylin-eosin. Scale bar $100 \mu \mathrm{m}$

Table 1. Assessment of quality of repair in human cartilage non interleukin- $1 \beta$-treated lesions (Non-IL) and ILI $\beta$-treated lesions (IL). International Cartilage Repair Society II (ICRS II) scale values for different scaffolds in the Non-ILI $\beta$-treated and ILI $\beta$-treated model. Our modified scoring system comprises 7 of the 14 original parameters.

\begin{tabular}{|c|c|c|c|c|c|c|c|c|}
\hline Parameter & $\begin{array}{l}\text { Col I } \\
\text { (Non-IL) }\end{array}$ & Col I (IL) & Col I +HS (Non-IL) & $\begin{array}{l}\text { Col I } \\
+\mathrm{HS} \\
(\mathrm{IL})\end{array}$ & $\begin{array}{l}\text { Col I + } \\
\text { Col II } \\
+ \text { HS } \\
\text { (Non-IL) }\end{array}$ & $\begin{array}{l}\text { Col I + } \\
\text { Col II } \\
+ \text { HS (IL) }\end{array}$ & $\begin{array}{l}\text { Col I } \\
+ \text { Col II +CHS } \\
(\text { Non-IL) }\end{array}$ & $\begin{array}{l}\mathrm{Col} \mathrm{I} \\
+\mathrm{Col} \mathrm{II} \mathrm{+CHS} \\
\text { (IL) }\end{array}$ \\
\hline Tissue morphology & 30 & 5 & 35 & 30 & 50 & 20 & 35 & 20 \\
\hline Matrix staining & 0 & 0 & 5 & 0 & 50 & 5 & 15 & 0 \\
\hline Cell morphology & 15 & 0 & 5 & 25 & 70 & 40 & 80 & 40 \\
\hline $\begin{array}{l}\text { Chondrocyte } \\
\text { clustering }\end{array}$ & 100 & 100 & 100 & 100 & 100 & 100 & 100 & 100 \\
\hline Surface architecture & 90 & 80 & 80 & 90 & 90 & 90 & 40 & 50 \\
\hline Integration & 30 & 5 & 60 & 50 & 80 & 65 & 10 & 50 \\
\hline Overall assessment & $44 \%$ & $31 \%$ & $47 \%$ & $49 \%$ & $73 \%$ & $53 \%$ & $46 \%$ & $43 \%$ \\
\hline
\end{tabular}

Col I: type I collagen; Col II: type II collagen; HS: heparan sulfate; CHS: chondroitin sulfat.

By macroscopic ICRS II assessment of the Non-IL model, the repair score was between $44 \%$ and $47 \%$, or fibrocartilage, for Col I, Col I+Col II+CHS and Col I+HS scaffolds. Col I+Col II+HS scaffolds showed a chondrogenic phenotype, $73 \%$, or mixed firbrocartilage and hyaline cartilage on the ICRS II scale. This was confirmed by histological and immunohistochemical analysis that showed the presence of PGs and Col, being positive for Col II and Agg (Figure 2).

\section{ILI $\beta$-treated model}

In the IL1 $\beta$ model, HE staining showed neotissue formation within the lesion with fewer rounded cells than in the Non-IL1 $\beta$ model (Figure 1, H-E IL). The presence of PGs detected by SO staining (Figure 1, $\mathrm{H}-\mathrm{E}$ IL) was more metachromatic in the $\mathrm{Col} \mathrm{I}+\mathrm{Col}$ II+CHS constructs $(23.228 \pm 1.704)$ than in Col I $(5.738 \pm 1.283)$, Col $\mathrm{I}+\mathrm{HS}(5.107 \pm 2.435)$ and $\mathrm{Col} \mathrm{I}+\mathrm{Col}$ II+HS (20.973 \pm 6.849$)$ constructs. These morphological changes with the loss of ECM components observed by SO and MT staining resulted in lowering of the ICRS II scores except those for the Col I+HS and Col I+Col II+CHS constructs $(49 \%-43 \%$, respectively) (Table 1).

\section{Discussion}

This model supposes an improvement compared with other 3D in vitro models.

Because cell phenotype and extracellular matrix (ECM) vary with cartilage depth [9], constructs, once implanted, are influenced by the native cell metabolism of each zone [10].

We found that cellular density in all the grafts was higher than in the native cartilage and, flattened-like undifferentiated cells were observed in all the scaffolds. The presence of undifferentiated hBMSCs or chondrocyte-like cells without zonal organization may be advantageous for Cartilage Engineering because immature tissue is capable of maturing when implanted in the joint [4].

Col I+Col II+HS scaffolds showed a fibrocartilage/hyaline cartilage phenotype, using the ICRS II scale. These results are similar to those obtained in a clinical study following microfracture treatment [11].

Chondrocytes implanted into focal or OA joint lesions were susceptible to native inflammation. Furthermore, problems with mechanics in OA and eventual loss of integrity, functionality and, long-term neotissue viability could be compromised [12]. Khan 
et al. found that a single short catabolic pulse of IL1 $\beta$ followed by post-culture anabolic conditions is sufficient to generate mechanically robust, integrative cartilage repair [13]. That is why secondly, an in vitro lesion model in which explants were pre-treated with IL1 $\beta$ before culture in chondrogenic medium was developed.

Different studies have shown that in vivo infiltration of IL1 $\beta$ into the joints results in degradation of Col and PGs from the chondrocyte ECM [13]. In our study, constructs showed lower amounts of PGs and less rounded cells after IL1 $\beta$ treatment than without treatment, except in CHS scaffolds. Scaffolds composed of CHS have been described as having better anti-inflammatory properties [14].

Regarding to the ICRS II scale, it was demonstrated lower inter- and intra-reader variability compared with other traditional scales. The overall assessment and matrix staining scores had the best correlation coefficients for inter- and intra-reader variability [8]. However, there are limitations of this study to take into account. It is necessary to consider any disadvantage of assessing the quality of repair in the in vitro developed models using the ICRS II scale, which was originally designed to evaluate in vivo osteochondral repair. ICRS II [8] was developed comprising 14 criteria: tissue morphology, matrix staining, cell morphology, chondrocyte clustering, surface architecture, basal integration, tidemark formation, subchondral bone fibrosis, inflammation, abnormal calcification, vascularization, surface assessment, mid/deep zone assessment and overall assessment. However, we focus on the assessment of 7 out of 14, which were related to chondrocyte phenotype and tissue structure. The assessment of the other 7 parameters was not possible in our developed model (tidemark formation, subchondral bone fibrosis, inflammation, abnormal calcification, vascularization, surface assessment and mid/deep zone assessment).

It is also relevant to point out that lack of mechanical stimuli negatively influences the quality of the neotissue formed. It is well known that continuous passive movement was shown to stimulate the repair of focal lesions with a neotissue similar to hyaline cartilage [9] and movement and loading in the joint were demonstrated to be essential for development and maintenance of normal cartilage structure [15]. The duration of our study was only two months, which is less time than that described in the literature. Because cartilage repair is a slow process, mathematical models have predicted that more than 18 months would be required to obtain complete cartilage maturation in vivo [5]. In vitro culture for long periods of time increases the risk of culture contamination and cartilage degradation [3]. However, our group had previously tested these two-month-culture-models without culture contamination or tissue degradation [16].

In conclusion, in an in vitro model using hBMSCs cultured on a Col I +Col II +HS scaffold displayed higher overall human cartilage lesion repair assessment than those of the other scaffolds. The capacity for repair by hBMSCs and the levels of construct chondrogenic phenotype of human cartilage lesions are lessened by pretreatment with IL1 $\beta$.

\section{Supplementary Material}

Figure S1. http:/ / www.medsci.org/v14p1257s1.pdf

\section{Abbreviations}

hBMSCs: human bone marrow mesenchymal stromal cells; Col: collagen; IL1 $\beta$ : interleukin $1 \beta ; \mathrm{Col}$ I: type I collagen scaffolds; Col I+HS: Col I and heparan sulfate scaffolds; Col I+Col II+HS: Col I, Col II and HS scaffolds; Col I+Col II+CHS: Col I, Col II and chondroitin sulfate scaffolds; TGF $\beta-3$ : transforming growth factor $\beta-3$; ICRS: International Cartilage Repair Society; 3D: three dimensional; OA: osteoarthritis; DMEM: medium Dulbecco's Modified Eagle's Medium; ECM: extracellular matrix; HE: hematoxylin and eosin; MT: Masson's trichrome; SO: Safranin O; PGs: proteoglycans; Agg: aggrecan.

\section{Acknowledgements}

Opocrin S.P.A. (Bruna Parma); laboratory technicians: María José Sánchez-Dopico, Purificación Filgueira-Fernández and Noa Goyanes-Rey from INIBIC-CHUAC for their support and assistance. This study was supported by grants: CAM (S2009/MAT-1472); CIBER-BBN, Instituto de Salud Carlos III (CB06/01/0040); Rede Galega de Terapia Celular and Grupos con Potencial de Crecemento, Xunta de Galicia (R2016/036, R2014/050 and GPC2014/048); MINECO-FEDER (RTC-2016-5386-1); Universidade da Coruña; Fundación Española de Reumatología (2014 grant); Fundación Profesor Novoa Santos and Diputación da Coruña. Clara Sanjurjo-Rodríguez and Rocío Castro-Viñuelas are beneficiaries of fellowships (postdoctoral and predoctoral, respectively) from Xunta de Galicia.

\section{Competing Interests}

The authors have declared that no competing interest exists.

\section{References}

1. Panadero JA, Lanceros-Mendez S, Gomez Ribelles JL. Differentiation of mesenchymal stem cells for cartilage tissue engineering: Individual and 
synergetic effects of three-dimensional environment and mechanical loading. ACTA BIOMATER. 2016; 33:1-12.

2. Chen WC, Yao CL, Wei YH et al. Evaluating osteochondral defect repair potential of autologous rabbit bone marrow cells on type II collagen scaffold. Cytotechnology. 2011;63(1):13-23.

3. Fuentes-Boquete IM, Arufe Gonda MC, Díaz Prado SM, et al. Cell and Tissue Transplant Strategies for Joint Lesions. Open Trans J. 2008; 2:21-28.

4. Pabbruwe MB, Esfandiari $\mathrm{E}$, Kafienah $\mathrm{W}$, et al. Induction of cartilage integration by a chondrocyte/collagen-scaffold implant. Biomaterials. 2009;30(26):4277-4286.

5. Lutianov M, Naire S, Roberts S, et al. A mathematical model of cartilage regeneration after cell therapy. J Theor Biol. 2011;289:136-150.

6. Sanjurjo-Rodríguez C, Martínez-Sánchez AH, Hermida-Gómez T, et al. Human Cartilage Tissue Engineering Using Type I Collagen/Heparan Sulfate Scaffolds. J Regen Med. 2014; 3 (2).

7. Sanjurjo-Rodríguez C, Martínez-Sánchez AH, Hermida-Gómez T, et al. Differentiation of human mesenchymal stromal cells cultured on collagen sponges for cartilage repair. Histol Histopathol. 2016;31(11):1221-1239.

8. Mainil-Varlet P, Van Damme B, Nesic D, et al. A new histology scoring system for the assessment of the quality of human cartilage repair: ICRS II. Am J Sports Med. 2010;38(5):880-890.

9. Newman AP. Articular cartilage repair. Am J Sports Med. 1998;26(2):309-324.

10. Negri S, Fila C, Farinato S, et al. Tissue engineering: chondrocyte culture on type 1 collagen support. Cytohistological and immunohistochemical study. J Tissue Eng Regen Med. 2007;1(2):158-159

11. Deng Z, Jin J, Zhao J, et al. Cartilage Defect Treatments: With or without Cells? Mesenchymal Stem Cells or Chondrocytes? Traditional or Matrix-Assisted? A Systematic Review and Meta-Analyses. Stem Cells Int. 2016;2016:9201492.

12. Wang D, Taboas JM, Tuan RS. PTHrP overexpression partially inhibits a mechanical strain-induced arthritic phenotype in chondrocytes. Osteoarthritis Cartilage. 2011;19(2):213-221.

13. Khan IM, Gonzalez LG, Francis $L$, et al. Interleukin-1 $\beta$ enhances cartilage-to-cartilage integration. Eur Cell Mater. 2011;22:190-201.

14. Taraballi F, Corradetti B, Minardi S, et al. Biomimetic collagenous scaffold to tune inflammation by targeting macrophages. J Tissue Eng. 2016;7:2041731415624667.

15. Khoshgoftar M, van Donkelaar CC, Ito K. Mechanical stimulation to stimulate formation of a physiological collagen architecture in tissue-engineered cartilage: a numerical study. Comput Methods Biomech Biomed Engin. 2011;14(2):135-144.

16. Muiños-López E, Hermida-Gómez T, Fuentes-Boquete I, et al. Human Amniotic Mesenchymal Stromal Cells as Favorable Source for Cartilage Repair. Tissue Eng Part A. 2017. 